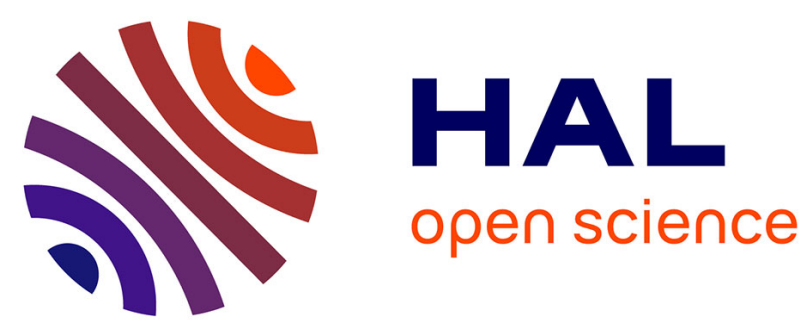

\title{
Exploring 1D and 2D Nanomaterials for Health Monitoring Wearable Devices
}

\author{
Gabriele Boschetto, Tieying Xu, Mohamad Yehya, Jérôme Thireau, Alain \\ Lacampagne, Benoit Charlot, Thierry Gil, Aida Todri-Sanial
}

\section{To cite this version:}

Gabriele Boschetto, Tieying Xu, Mohamad Yehya, Jérôme Thireau, Alain Lacampagne, et al.. Exploring 1D and 2D Nanomaterials for Health Monitoring Wearable Devices. FLEPS 2021 - IEEE International Conference on Flexible and Printable Sensors and Systems, Jun 2021, Virtual, United Kingdom. pp.1-4, 10.1109/FLEPS51544.2021.9469864 • lirmm-03363641

\section{HAL Id: lirmm-03363641 https://hal-lirmm.ccsd.cnrs.fr/lirmm-03363641}

Submitted on 5 Oct 2021

HAL is a multi-disciplinary open access archive for the deposit and dissemination of scientific research documents, whether they are published or not. The documents may come from teaching and research institutions in France or abroad, or from public or private research centers.
L'archive ouverte pluridisciplinaire HAL, est destinée au dépôt et à la diffusion de documents scientifiques de niveau recherche, publiés ou non, émanant des établissements d'enseignement et de recherche français ou étrangers, des laboratoires publics ou privés. 


\title{
Exploring 1D and 2D Nanomaterials for Health Monitoring Wearable Devices
}

\author{
Gabriele Boschetto*, Tieying Xu ${ }^{\dagger}$, Mohamad Yehya ${ }^{\ddagger}$, Jerome Thireau ${ }^{\ddagger}$, Alain Lacampagne ${ }^{\ddagger}$, Benoit Charlot $^{\dagger}$, \\ Thierry Gil* and Aida Todri-Sanial ${ }^{*}$ \\ *LIRMM, Université de Montpellier, CNRS, Montpellier 34095, France \\ Email: aida.todri@lirmm.fr \\ ${ }^{\dagger}$ IES, Université de Montpellier, CNRS, Montpellier 34090, France \\ Email: benoit.charlot@um2.fr \\ †PhyMedExp, Université de Montpellier, CNRS, INSERM, Montpellier 34295, France \\ Email: alain.lacampagne@inserm.fr
}

\begin{abstract}
In this work we explore 1D and 2D nanomaterials (carbon nanotubes and $\mathrm{MoS}_{2}$, respectively) as the core sensing components of novel highly sensitive and flexible strain sensors to monitor patients' vital signs, such as respiratory rate. We assess their performance following a multi-disciplinary and multi-level approach, starting from atomistic simulations, up to device fabrication and applications. By successfully designing, fabricating, and testing a flexible strain sensor capable of tracking the respiratory rate, we show that these materials are excellent candidates to be used for the development of next generation health monitoring wearable devices.
\end{abstract}

Index Terms-Wearable sensors, Nanomaterials, Carbon nanotubes, Molybdenum disulfide $\left(\mathrm{MoS}_{2}\right)$, Density functional theory (DFT), Internet-of-Medical-Things (IoMT)

\section{INTRODUCTION}

In recent years, the discovery and synthesis of novel (nano)materials and the development of new fabrication techniques have enabled the rapid evolution of flexible electronic technologies, with numerous applications in areas such as energy harvesting (e.g., photovoltaics), robotics, display devices (flexible OLEDs), and healthcare [1].

In the context of Internet of Medical Things (IoMT) [2], wearable strain sensors based on flexible materials and architectures could be well-suited to be employed for the continuous and non-intrusive measurement of biosignals such as heart and respiratory rates, body temperature, and blood pressure. To be used effectively, such sensors have to well adhere to the patient's skin, be highly stretchable and flexible in order to follow the patient's movements, be ultra sensitive, and, importantly, be biocompatible. In order to meet these requirements, the good choice of the sensing materials is pivotal, as conventional devices based on metals and silicon are not much bendable and generally not capable to withstand large applied strain. To overcome such limitations, we explored low-dimensional materials [3], [4], such as carbon nanotubes (CNTs) and transition metal dichalcogenides (TMDs), as these could potentially offer both high stretchability and high sensitivity.

This project has received funding from the European Union's Horizon 2020 research and innovation programme, EU H2020 SmartVista (www.smartvista.eu), grant agreement No. 825114.
Thus, in this work we assess the fitness for purpose of CNT nanocomposites and molybdenum disulfide $\left(\mathrm{MoS}_{2}\right)$ for the development of stretchable strain sensors to monitor patients' respiratory rate. To do so, we followed a multi-disciplinary and multi-level approach, tackling the problem from different perspectives: i) at the atomic scale via first principles atomistic simulations; ii) at the device level (fabrication and characterization); iii) in real applications, with ex-vivo experiments. Our "holistic" approach allowed us to better understand the complex physics of these nanomaterials when subject to strain and to develop a CNT flexible strain sensor which is able to capture and track the respiratory function of living beings.

\section{Design of the Strain Sensor}

\section{A. Materials and Modelling}

In this part of the work we performed density functional theory (DFT) simulations to elucidate the physics of both CNT networks and $\mathrm{MoS}_{2}$ when subject to strain. Moreover, electronic structure theory provides fundamental information to predict relevant materials' parameters, such as band gap and electron mobility. These simulations lay the necessary theoretical foundation needed for advancing the device optimization.

We first focused on CNTs as these are excellent candidates to be used in flexible devices, given their high mechanical flexibility and intrinsic charge carrier mobility. Moreover, compared to metallic strain gauges, CNT networks possess high gauge factors, high sensitivity, stability, and reliability [5], [6]. The change in the resistivity could be explained by considering the percolation theory: CNTs can form connective networks when the intertube distance is small enough to create a close contact (i.e., a junction). When strain is applied, it is expected that such close contacts will break while others will form, leading to a significant change in the overall network resistivity. We point out that most ab initio theoretical studies focused on modelling strain by elongating (or compressing) the $\mathrm{C}-\mathrm{C}$ bonds within a single CNT [7], rather than modelling strain in a network.

Equally promising to be used in sensing devices is monolayer $\mathrm{MoS}_{2}$. In particular, the high tunability of its unique electronic and mechanical properties [8], and its high charge 


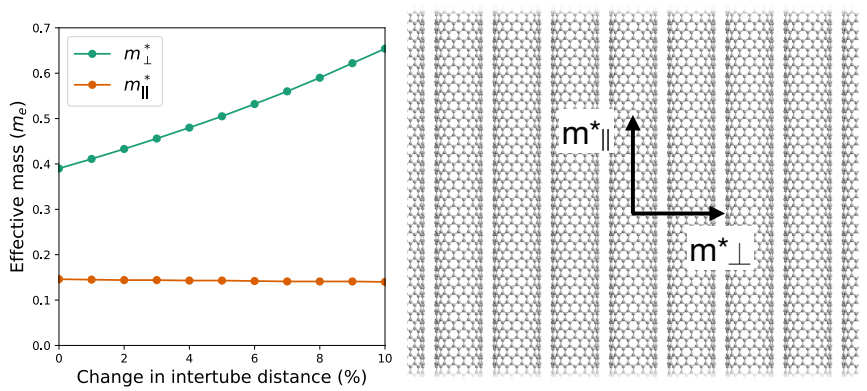

Figure 1. Plot of the electron effective mass of the $(14,0)$ CNT network as a function of strain, computed with DFT both along $\left(m_{\|}^{*}\right)$ and across $\left(m_{\perp}^{*}\right)$ the nanotubes. A representation of the CNT network with the relevant directions along which the effective mass were computed is also shown.

carrier mobility [9], have pushed the material to be investigated for the use in numerous nanodevices, including biosensors [10].

Atomistic simulations were carried out within the density functional theory (DFT) framework by using QuantumATK software [11], [12]. To model core electrons, we used normconserving pseudopotentials from the PseudoDojo library [13]. All simulations were carried out in vacuum using the PerdewBurke-Ernzerhof (PBE) exchange-correlation functional [14] and, to correctly predict the intertube distance, we included the D2 correction by Grimme [15] to take into account dispersion effects (PBE-D2). We overcame the challenge of modelling CNT networks with DFT by considering a series of infinitely aligned and parallel semiconducting $(14,0) \mathrm{CNTs}$, placed at the equilibrium intertube distance of $3.07 \AA$, which we obtained after relaxing the atomic coordinates of a model $(14,0)$ CNT dimer. We note that our result is consistent with previous studies in the literature [16]. For CNTs we defined strain as the change in the intertube distance, which we have progressively increased. We modelled $\mathrm{MoS}_{2}$ as a monolayer, and we considered an orthogonal simulation cell, of which we have fully optimised both the atomic coordinates and the lattice vectors. We applied both uniaxial (along the zigzag, $x$, and the armchair, $y$, directions) and equibiaxial strain, which we defined as the change in the lattice vectors. For both materials, we estimated electron mobilities by computing the effective masses along relevant directions, taking the values of the band curvature at the conduction minima of the materials' band structures.

Our simulations of CNT networks allowed us to decompose the average network mobility onto two orthogonal directions, i.e. along $\left(m_{\|}^{*}\right)$ and across $\left(m_{\perp}^{*}\right)$ the nanotubes. Results are shown in Figure 1, in which we plotted the effective masses computed along both directions against the change in the equilibrium intertube distance: interestingly, we observe low values $\left(<1 m_{e}\right)$ along both directions, thus we expect good mobilities not only within single nanotubes, but also across the intertube close contacts. We also note that, at the equilibrium distance, $m_{\perp}^{*}$ is only 2-3 times larger than $m_{\|}^{*}$. As we apply increasing strain up to $10 \%, m_{\|}^{*}$ remains constant, since the

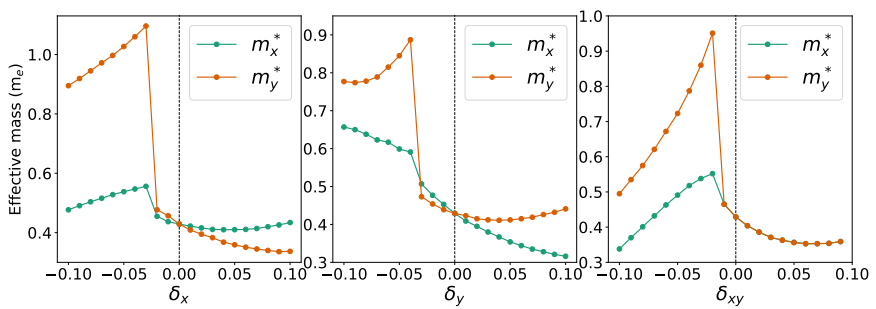

Figure 2. Plot of the electron effective mass of $\mathrm{MoS}_{2}$ as a function of strain computed along the zigzag $\left(m_{x}^{*}\right)$ and armchair $\left(m_{y}^{*}\right)$ directions. $\delta_{x}$ and $\delta_{y}$ correspond to uniaxial strain applied along the zigzag and armchair directions, respectively, whereas $\delta_{x y}$ corresponds to equibiaxial strain.

chemical structure of the nanotubes remained unchanged. On the contrary, $m_{\perp}^{*}$ progressively increases with the increase in strain, i.e. when close contacts get broken, consistently with the percolation theory. We also computed the band gap of the CNT network and we observed a rather small increase $(+0.1 \mathrm{eV}$ at $10 \%$ strain), suggesting that varying the intertube distance has very little effect on this quantity.

We then applied both tensile and compressive strain (between $-10 \%$ and $10 \%$ ) on monolayer $\mathrm{MoS}_{2}$, and at each time we computed the effective masses along the orthogonal zigzag $\left(m_{x}^{*}\right)$ and armchair $\left(m_{y}^{*}\right)$ directions. As can be seen from Figure 2, without any applied strain both $m_{x}^{*}$ and $m_{y}^{*}$ have the same value, suggesting that the mobility in the unstrained material is isotropic. However, if directional strain is applied, $\mathrm{MoS}_{2}$ shows anisotropic mobility, with a significant variability in the case of compressive strain. When equibiaxial strain is applied, we observe that the material retains its isotropic behavior if subject to tensile strain, whereas compressive strain once again leads to anisotropy. Moreover, we also observed a significant variability $(>1 \mathrm{eV})$ in the band gap of the material; notably, at $10 \%$ tensile equibiaxial strain we noticed the vanishing of this quantity, with $\mathrm{MoS}_{2}$ becoming metallic. This outstanding tunability of its electronic properties reinforces the idea that $\mathrm{MoS}_{2}$ could be successfully employed for ultra sensitive strain devices.

\section{B. Fabrication and Characterization}

Here, we describe the fabrication process of our CNT strain sensor. We point out that although, as previously mentioned, CNT networks offer significant advantages over metallic materials, in nanocomposites the elasticity of the polymer encapsulation matrix is equally crucial for the overall flexibility of the wearable strain sensor. Therefore, we chose Dragon Skin silicone because of its high deformability and its biocompatibility. In the first step, the Dragon Skin encapsulation was moulded from a macroscale filament substrate. The groove of CNTs was realized by 3D printing technique. Part A ( $5 \mathrm{~g})$ and part B (5 g) of Dragon Skin were mixed in a beaker for 2 minutes before degassing. The mixture was then poured into the filament mold with the groove pattern, and cured in the oven for 10 minutes. Eventually, the Dragon Skin bottom layer was removed from the filament to allow the injection of CNTs. In the second step, $5 \mathrm{~g}$ of CNTs powder was mixed with 50 


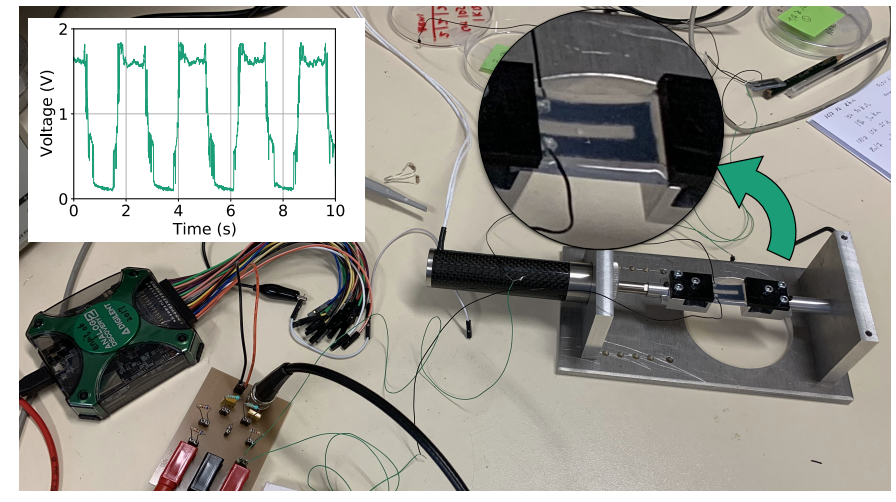

Figure 3. Experimental setup and characterization curve of the CNT-based strain sensor, which is shown at the upper right corner of the figure.

$\mathrm{ml}$ of acetone, following a sonication process for 1 hour to unchain the CNTs. Afterwards, $50 \mu \mathrm{l}$ of the CNT solution were injected each time into the groove of Dragon Skin. We repeated this step 5-6 times until we observed the formation of a homogeneous CNT network. In the final step, the copper wire was fixed at both sides of the groove with silver paste. Finally, the CNT network and the bottom layer of the Dragon Skin were both encapsulated by a top layer of Dragon Skin.

The CNT/DS strain sensor was tested by using the E-861 PiezoWalk ${ }^{\circledR}$ NEXACT ${ }^{\circledR}$ Controller by varying strain at rates ranging between 0.01 and $10 \mathrm{~mm} / \mathrm{s}$. The resistance of the sensor was equal to $20 \mathrm{k} \Omega$. A homemade Wheatstone bridge circuit structure was fabricated by using known resistances equal to $22 \mathrm{k} \Omega$. The supply DC voltage $V_{c c}$ was set to $1.8 \mathrm{~V}$ at the stretch rate of $0.5 \mathrm{~mm} / \mathrm{s}$, and, as the electrical circuit balance was broken, the induced current was detected and transformed into voltage signal. Such signal was recorded by Analog Discovery and the signal evolution curve is shown in Figure 3, together with our experimental setup. The curve shows that our CNT sensor is sensitive to strain, and that its response is reproducible over time.

\section{EX-VIVO TESTING OF RESPIRATORY FUNCTION}

Male Wistar rats were housed on a twelve-hour light-dark cycle and had free access to water and food. All investigations conformed to European Parliament Directive 2010/63/EU and were approved by the local ethics committee rules (Ethics committee for animal experimentation Languedoc-Roussillon $\mathrm{N}^{\circ}$ 22699-2019110611232613 v3).

All experiments were carried out on anesthetized rats (isoflurane $2.5-3 \%$ ). The CNT strain sensor was attached to the rat's chest skin in a number of ways: either using crocodile clips or tape, or sewn directly to the skin. While the strain sensor was stretching, the resistance value $\left(R_{\text {sensor }}\right)$ change, due to the mechanical deformation induced by respiration, was detected and transformed into voltage signal. Voltage signal variation was monitored at first by using the microcontroller board and STM studio hardware, and then with Analog Discovery 2 (a multifunction hardware) and the WaveForms software. By using Analog Discovery 2, the fast

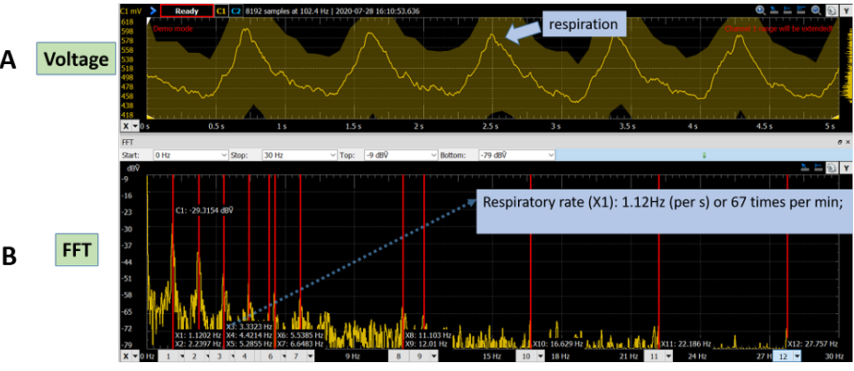

Figure 4. Voltage signal curve in monitoring the respiration of an anesthetized rat. A) Peaks of voltage signal variation which correspond to breath cycles. B) FFT value in $\mathrm{Hz}$ corresponding to the number of breathing cycles per second or per minute by multiplying the value by 60 .

Fourier transform (FFT) was obtained at the same time in addition to the output voltage signal curve.

We performed different experiments, and at first the strain sensor was attached to the anesthetized animal's skin with crocodile clips. We manually counted, during one minute, the rat's breath cycles and the number of peaks shown on the STM studio software. We found a corresponding number of 55 breath cycles per minutes (bpm) and 55 peaks recorded on the software. In a configuration similar to that described previously, we monitored once again the variation of the voltage signal but using Analog Discovery 2 hardware and the WaveForms software. We found a correspondence between the number of respiratory cycles manually counted and the number of peaks displayed by the software at the same time: 67 breath cycles with FFT $1.13 \mathrm{~Hz}$ (see Figure 4). In the final experiment, the CNT strain sensor was sewn directly to the rat's chest skin. In accordance with previous experiments, we found the same correspondence between the number of breath cycles and the number of voltage peaks recorded by the software: 24 breath cycles were recorded during 30 seconds and 24 peaks were measured by the software, which correspond to FTT $0.8 \mathrm{~Hz}$.

\section{CONCLUSIONS}

We successfully designed, fabricated, and tested a CNTbased strain sensor able to monitor the respiratory rate, and we showed that both 1D and 2D nanomaterials are very promising candidates to be used for such application. Stretchable strain sensors could play a vital role in monitoring patients with cardiovascular diseases as well as their breathing and heart rate. For example, the monitoring of respiratory rate has become increasingly essential in several healthcare settings for conditions such as sleep apnea and heart failure. Furthermore, the applications of this medical surveillance device are extremely promising, as it could be deployed in the service of patients with COVID-19 in the near future. As upcoming work, following the results of our simulations, we aim at fabricating and testing $\mathrm{MoS}_{2}$-based strain sensors to monitor both heart and respiratory rates. We are also interested in further investigating the properties of both nanomaterials by increasing the complexity of our theoretical models, for instance by including non-uniform strain. 


\section{REFERENCES}

[1] M. Zou, Y. Ma, X. Yuan, Y. Hu, J. Liu, and Z. Jin, "Flexible devices: from materials, architectures to applications," J. Semicond., vol. 39, p. 011010, 2018

[2] C. A. da Costa, C. F. Pasluosta, B. Eskofier, D. Bandeira da Silva, and C. da Rosa Righi, "Internet of health things: Toward intelligent vital signs monitoring in hospital wards," Artif. Intell. Med., vol. 89, pp. 6169, 2018.

[3] R. Mas-Ballesté, C. Gómez-Navarro, J. Gómez-Herrero, and F. Zamora, " $2 \mathrm{~d}$ materials: to graphene and beyond," Nanoscale, vol. 3, pp. 20-30, 2011.

[4] E. Garnet, L. Mai, and P. Yang, "Introduction: 1d nanomaterials/nanowires," Chem. Rev., vol. 119, pp. 8955-8957, 2019.

[5] E. W. Wong, P. E. Sheehan, and C. M. Lieber, "Nanobeam mechanics: Elasticity, strength, and toughness of nanorods and nanotubes," Science, vol. 277, pp. 1971-1975, 1997.

[6] M. Zhang, K. R. Atkinson, and R. H. Baughman, "Multifunctional carbon nanotube yarns by downsizing an ancient technology," Science, vol. 306, pp. 1358-1361, 2004.

[7] C. Wagner, J. Schuster, and T. Gessner, "DFT investigations of the piezoresistive effect of carbon nanotubes for sensor application," Phys. Status Solidi, vol. 249, pp. 2450-2453, 2012.

[8] H. J. Conley, B. Wang, J. I. Ziegler, R. F. Haglund, S. T. Pantelides, and K. I. Bolotin, "Bandgap engineering of strained monolayer and bilayer $\mathrm{MoS}_{2}$," Nano Lett., vol. 13, pp. 3626-3630, 2013.

[9] Z. Yu, Z.-Y. Ong, L. S., J.-B. X, G. Zhang, Y.-W. Zhang, Y. Shi, and $\mathrm{X}$. Wang, "Analyzing the carrier mobility in transition-metal dichalcogenide $\mathrm{MoS}_{2}$ field-effect transistors," Adv. Funct. Mater, vol. 17, p. 1604093, 2017.

[10] S. Barua, H. S. Dutta, S. Gogoi, R. Devi, and R. Khan, "Nanostructured $\mathrm{MoS}_{2}$-based advanced biosensors: A review," ACS Appl. Nano Mater, vol. 1 , pp. $2-25,2018$

[11] S. Smidstrup, T. Markussen, P. Vancraeyveld, J. Wellendorff, J. Schneider, T. Gunst, B. Verstichel, D. Stradi, P. A. Khomyakov, U. G. VejHansen et al., "QuantumATK: An integrated platform of electronic and atomic-scale modelling tools," J. Phys: Condens. Matter, vol. 32, p. 015901, 2020

[12] QuantumATK version R-2020.09, Synopsys QuantumATK (www.synopsys.com/silicon/quantumatk.html).

[13] M. J. van Setten, M. Giantomassi, E. Bousquet, M. J. Verstraete, D. R. Hamann, X. Gonze, and G.-M. Rignanese, "The PseudoDojo: Training and grading a 85 element optimized norm-conserving pseudopotential table," Comput. Phys. Commun., vol. 226, pp. 39-54, 2018.

[14] J. P. Perdew, K. Burke, and M. Ernzerhof, "Generalized gradient approximation made simple," Phys. Rev. Lett., vol. 77, pp. 3865-3868, 1996.

[15] S. Grimme, "Semiempirical GGA-type density functional constructed with a long-range dispersion correction," J. Comput. Chem., vol. 27, pp. 1787-1799, 2006.

[16] A. Szabados, L. P. Biró, and P. R. Surján, "Intertube interactions in carbon nanotube bundles," Phys. Rev. B, vol. 73, p. 195404, 2006. 